\title{
"Other" indirect methods for nuclear astrophysics
}

\author{
Livius Trache \\ IFIN-HH, Bucharest-Magurele, Romania
}

\begin{abstract}
In the house of Trojan Horse Method (THM), I will say a few words about "other" indirect methods we use in Nuclear Physics for Astrophysics. In particular those using Rare Ion Beams that can be used to evaluate radiative proton capture reactions. I add words about work done with the Professore we celebrate today. With a proposal, and some results with TECSA, for a simple method to produce and use isomeric beam of ${ }^{26 \mathrm{~m}} \mathrm{Al}$.
\end{abstract}

\section{Introduction}

As this is an invited lecture in the special session dedicated to prof. Claudio Spitaleri on the occasion of his (formal) retirement, I will treat it just like that! I mean, I will keep the promise of the title and abstract, but I will insist on work done together with Claudio and his people. It will obviously be difficult to put in 6 pages the content of 35 slides, including images worth 1000 words each! In all cases I will enumerate, rather than fully describe or justify, the most common indirect methods that we use in nuclear astrophysics. In this age when nuclear physics for astrophysics (NPA) and the rare ion beams (RIB) are spearheading most nuclear physics programs, the combination of the two is done through indirect methods (so far at least). That is, by using measurements at laboratory energies of tens or even hundreds or $\mathrm{MeV} /$ nucleon to obtain data that allow us to evaluate cross sections and/or reaction rates for nuclear processes that take place in stars at energies of tens - hundreds of $\mathrm{keV} /$ nucleon, or even lower. I cannot pretend in such a short time (during the conference) and space (in the Proceedings) to cover exhaustingly all indirect methods and all what was done so far with them. Therefore I will be very selective and subjective and in all cases I will use examples from works I participated to and which, to one extent or another, can be related to the honouree of the session. In particular in Sec. 3 and 4, where I will present TECSA and one interesting use of it, respectively.

I met Professore Spitaleri sometime last century! And as many of you here, I met him first through a paper of his before meeting him in person. That first encounter was the origin of many works we did, or we participated to, together, either at Texas A\&M University, in Catania, or at IFIN-HH in Bucharest. Certainly others in this session and this room know him better and for longer, and they already have spoken or will, and said already moving words about him and his works. Repeating those will only seem formal. But I say that it is important to tell from the beginning that when I think and talk about Claudio I always have in mind also his group of people. A group that he raised in Catania, 
at the University and/or at LNS, which most probably represent his best and most enduring achievement of the career. Congratulations for that!

\section{Indirect Methods in Nuclear Astrophysics}

It is well known that we need to use indirect methods in NA basically for two reasons:

a) Stars are "cold" and the corresponding cross sections for the nuclear reactions in stars or stellar environments are very small when charged particles are involved, due to the Coulomb barriers;

b) Most of the reactions in stars involve radioactive species, which do not exist in nature, but can be produced in laboratory.

Many measurements in NPA are not direct measurements (= reactions between partners at stellar energies or close to them), are therefore indirect, but one cannot always classify easily them in a few types. Therefore the list of types of indirect methods may be subjective. Mine reads like this:

A. Coulomb dissociation

B. Transfer reactions (the ANC method)

C. Breakup (nuclear) of loosely bound nuclei

D. Resonance spectroscopy: $\beta$-decay, $\beta$-delayed proton-decay, transfer reactions, resonant elastic scattering, etc.

E. Trojan Horse Method.

\subsection{Coulomb Dissociation}

The study of a radiative capture reaction can be replaced by measuring its inverse, the photo-dissociation of the final nucleus. Coulomb dissociation (CD) is the process in which photo-dissociation is produced by virtual, rather than real photons [1]. One of the initiators of $\mathrm{CD}$ is in the audience, can better describe it, several other talks were describing it. I want only to comment on the fact that CD is sometimes called "the first indirect method in NA". I think that we had other indirect methods before. For example the study of weak interactions that allowed Bethe to evaluate the first step in the pp-chains. Or even, the mass measurements that allowed Sir A. Eddington to suggest that nuclear forces are at the origin of the energy production in Sun and stars!

\subsection{Transfer reactions (the ANC method)}

Transfer reactions were crucial in obtaining information about the structure of nuclei, in particular about their single particle, fermionic, structure. For over two decades now it was demonstrated and is recognized that one can use peripheral one-proton [2] or even oneneutron [3] transfer reactions to extract information about the peripheral behaviour of the radial wave function of the least bound nucleon and from it to evaluate the direct (nonresonant) component of the radiative proton capture reactions at low energies. The method consists in measuring cross sections for the transfer of a proton in nucleus-nucleus reactions, which allow the determination of the Asymptotic Normalization Coefficient (ANC) through comparison with DWBA calculations. It is applicable if the reaction is peripheral and one-step, and if good optical model potentials are available. The group at Texas A\&M University was instrumental in proposing using and demonstrating its validity. It was applied to stable and radioactive beams, as well [4, 5, 6 and references therein]. It was extended to radiative alpha capture too (examples in $[7,8]$ ). 


\subsection{Breakup (nuclear) of loosely bound nuclei}

The nuclear breakup of loosely bound nuclei can also be peripheral. In a reaction of the type $\mathrm{Y} \rightarrow \mathrm{X}+\mathrm{p}$ (breakup of a proton) at intermediate energies, from the shape of the measured momentum distribution of the core one can extract information about the quantum numbers of the proton orbital involved and from the cross section one can extract the ANC by comparison with appropriate calculations. Which ANC in turn can be used to evaluate the radiative capture cross section for the reaction $\mathrm{X}(\mathrm{p}, \gamma) \mathrm{Y}$. We applied it successfully in several cases $[9,10]$. I would argue that in many cases its most significant contribution was that cross sections (or astrophysical S-factor) where determined in reactions of crucial importance through a method that implied totally different systematic errors than those determined directly. Like in the case of the $\mathrm{S}_{17}(0)$-factor), where we could extract $\mathrm{S}_{17}(0)=18.7(19) \mathrm{eVb}$ [9], in good agreement with the best direct measurements for the ${ }^{7} \mathrm{Be}(\mathrm{p}, \gamma)^{8} \mathrm{~B}$ reaction, of crucial importance for the understanding of the solar neutrino puzzle.

\subsection{Resonance spectroscopy: $\beta$-decay, $\beta$-delayed proton-decay, transfer reactions, elastic scattering, etc.}

In several cases reactions at stellar energies are proceeding through, and are even dominated by resonances. The reaction is a two-step process in this case: a meta-stable state in the compound nucleus is populated in the entrance channel, which later decays to the final configuration. In these situations, the reaction rates get sizable contributions from the resonances that are located approximately in the Gamow window of the reaction. The contribution of each isolated narrow resonance is entirely dependent on two parameters: the location of the resonance (its energy) and the resonant strength. If both (or at least one, the location for example) can be determined by the spectroscopy of the metastable state that constitutes the resonance, then we can evaluate the contribution to the reaction rate. In many cases it is easier to determine the position of the resonance, and this is important as it affects exponentially the rate. Any spectroscopic method that would then allow to find these resonances and characterize them, is an indirect method. One with an obvious connection is the beta-delayed proton decay: if conditions allow it $\left(Q_{\beta}>S_{p}\right.$ and $\beta$-decay selection rules allow for populating same states relevant in proton capture), the metastable state may decay by proton emission; measured, it can determine its location, quantum numbers and decay branchings. There are two experimental problems we encounter, though: (1) the low proton energies relevant in NA are difficult to measure experimentally and (2) the lower the energy of protons, the lower the proton branching (due to a wider Coulomb barrier to be penetrating out). The combinations of these factors lead us to try first to detect $\beta$ p-decay using very thin Si detectors (65 and 45 microns, see [11]), not quite satisfactory, as there was still a large beta-induced background at low energies. Another idea was to use a gas detector, based on a novel type of detector with Micromegas as amplifier. This was much better, detectors named ASTROBOX1 and ASTROBOX2 gave good results $[12,13]$ and the idea is already spreading (see these Proceedings).

\subsection{Trojan Horse Method}

This is a Catania speciality and Claudio's role here is crucial! You have heard much about it, I do not believe I have anything to add at this point. Except, may be, a comment: it was proposed about 30 years ago. And it produces excellent results for more than two decades, but no other group was so far able to adopt it fully and produce independent experimental results! One should do that in the next future, for the good of the recognition of the method. 
And also say that it is the method that gives results that look closer to those of direct measurements: look in these Proceedings at $\mathrm{S}$-factor graphs going down to $\mathrm{E}=0$, and even under the threshold! Plus its first use with radioactive beams for the study of the ${ }^{18} \mathrm{~F}(\mathrm{p}, \alpha){ }^{15} \mathrm{O}$ reaction $[14,15]$.

\section{TECSA - Texas-Edinburgh-Catania Silicon Array}

At some point in the cooperation with Claudio and his group, we realized that we can put together a device by employing existing Texas A\&M facility (MARS) and manpower, complex multi-channel electronics from the Edinburgh group (PJ Woods, T. Davidson) and Catania's YY1 Si detectors. TECSA was built without any extra funding and a number of experiments were done at TAMU with it, some being "firsts" in their class. The commissioning was a ${ }^{14} \mathrm{C}(\mathrm{d}, \mathrm{p})$ experiment in inverse kinematics, using ${ }^{14} \mathrm{C}$ radioactive beam accelerated by the $\mathrm{K} 500$ superconducting cyclotron onto a $\mathrm{CD}_{2}$ target $[16,17]$. In Figure 1 bellow, I show online data: the right panel lets you understand the geometry of the array, the left one shows the population of ground and first excited state in ${ }^{15} \mathrm{C}$ (with good hints on angular distributions).

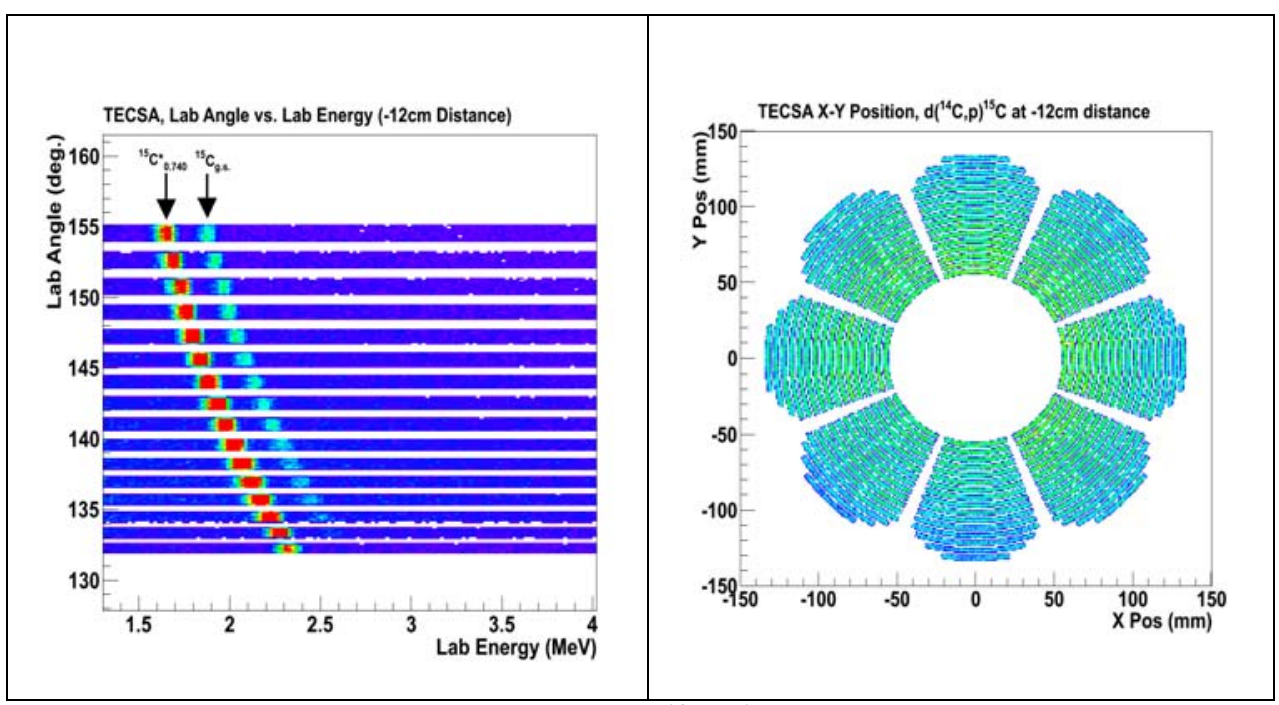

Figure 1. Data from the reaction $\mathrm{d}\left({ }^{14} \mathrm{C}, \mathrm{p}\right){ }^{15} \mathrm{C}$ taken with TECSA.

TECSA was also used for the study of the first THM using radioactive beam: d(18F,p)n15O.

\section{$4{ }^{26 m} \mathrm{Al}$ beam production: proposal and demonstration}

I will treat specially this experiment, which was the last before we dismantled TECSA in the spring of 2012. It is about obtaining am isomeric beam using a production mechanism, rather than a separator.

The isotope ${ }^{26} \mathrm{Al}$ is unstable with a long half-life of 0.72 million years for its $\mathrm{J}=5^{+}$ ground state and has an isomeric state $\mathrm{J}=0^{+}$only $228 \mathrm{keV}$ above with a much shorter halflife of $6.34 \mathrm{sec}$. The isotope is known to be important in nuclear astrophysics: the gammaray line $\mathrm{E} \gamma=1809 \mathrm{keV}$ following the beta-decay of its long-lived ground state was the first discrete line to be detected by gamma-ray satellite telescopes. This was a proof of on-going 
nucleosynthesis in the Universe. The origin of freshly produced ${ }^{26} \mathrm{Al}$ is not well known, however, and the comparison of observations with nucleosynthesis model predictions is hindered by the lack of precise nuclear data for some reactions in the reaction networks used in calculations. For all temperatures below $2 \mathrm{GK},{ }^{26 \mathrm{~g}} \mathrm{Al}$ and ${ }^{26 \mathrm{~m}} \mathrm{Al}$ behave like separate, different isotopes and are to be treated as such in calculations. For higher temperatures, only reached in some explosive scenarios, they will mix. Therefore, it is important to evaluate reactions on ${ }^{26 \mathrm{~m}} \mathrm{Al}$ and those can only be studied experimentally with an isomeric beam. The two states, g.s. and isomer, cannot be separated by current mass separators, because resolutions of $228 \mathrm{keV}$ at masses of about $26 \mathrm{GeV}$ are not available at current accelerators. Different methods must be found. We tried the use of a selective reaction mechanism at the MARS separator of the Cyclotron Institute of Texas A\&M University. We started with a ${ }^{26} \mathrm{Mg}$ primary beam of $16 \mathrm{MeV} /$ nucleon from the superconducting cyclotron $\mathrm{K} 500$ bombarding a $\mathrm{H}_{2}$ cryotarget. With it ${ }^{26} \mathrm{Al}$ could be produced by ${ }^{26} \mathrm{Mg}(\mathrm{p}, \mathrm{n})$ reaction by two mechanisms: fusion-evaporation and charge-exchange. The first should not be selective and produce a mixing of g.s. $\left(\mathrm{J}=5^{+}\right)$and isomeric state $\left(\mathrm{J}=0^{+}\right)$around the velocity of the compound system, while charge-exchange should select the isomeric state through the GT transition: $\left(v \mathrm{~d}_{5 / 2} * v \mathrm{~d}_{5 / 2}\right) 0^{+} \rightarrow\left(v \mathrm{~d}_{5 / 2} * \pi \mathrm{d}_{5 / 2}\right) 0^{+}$at velocities close to that of the projectile. By tuning MARS for the appropriate magnetic rigidities we did separate the two. When we tuned MARS for the fusion-evaporation products we obtained a pure ${ }^{26} \mathrm{Al}$ beam of $3 * 10^{6}$ pps with $33 \%{ }^{26 \mathrm{~m}} \mathrm{Al}$ content. When we changed the rigidity to that of the transfer reaction, we increased the ${ }^{26 \mathrm{~m}} \mathrm{Al}$ content of the beam to $67 \%$, but the intensity decreased 10 times to about $3 * 10^{5}$ pps. The intensities were sufficient in both cases to study $(\mathrm{d}, \mathrm{p})$ reactions in inverse kinematics using TECSA detection system. The comparison of the results show that the two beams can populate same or different excited states in the final nucleus ${ }^{27} \mathrm{Al}$. Details of the experiment and the results were only presented at one meeting and published online in the report [18], but it occurred to me that the method works and could be used further (Note added in Proceedings: it was pointed to me that in a paper published in August this year, an ANL group reported a similar idea [19]). It follows from the experiment that the production of the isomeric beam using a selective population mechanism like charge-exchange is possible. A better isomeric purity can be obtained by increasing the bombarding energy of the primary beam to $25-40 \mathrm{MeV} /$ nucleon. The isomer production through charge-exchange will be favored by the larger energy and at the same time the fusion-evaporation mechanism will lead mostly to ${ }^{25} \mathrm{Al}$ through $(\mathrm{p}, 2 \mathrm{n})$. Also the rigidity separation between the two components will increase, favoring a better separation.

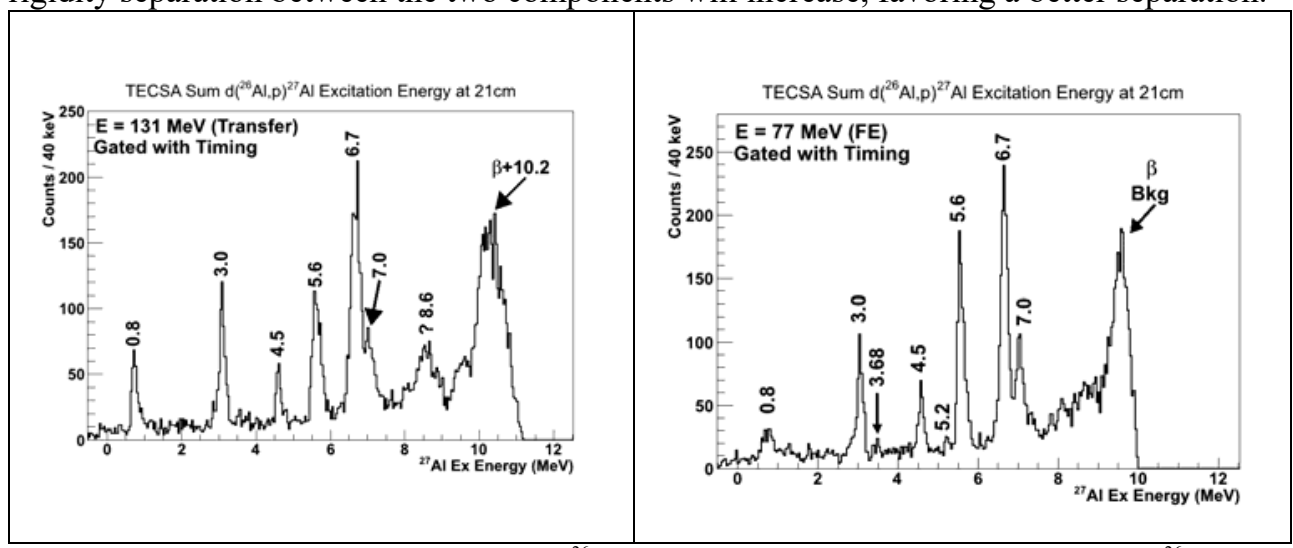

Figure 2. Part of the (d,p) spectra obtained with ${ }^{26 \mathrm{~m}} \mathrm{Al}$ beam $67 \%$ composition (left panel) and with ${ }^{26 \mathrm{~g}} \mathrm{Al}$ beam (right). Radioactive beams produced and separated with MARS, spectra taken with TECSA (2012). 


\section{Laudatio}

I want to close by a few words as laudatio for Claudio! Best laudatio in this case is to enumerate a few of his achievements. Trojan Horse Method was invented, demonstrated, used, spread to over 10 laboratories where Claudio and his group had experiments. Nuclear astrophysics has become a recognized mark of Catania laboratories. Claudio is appreciated and has a large number of friends in the global nuclear astrophysics community. He is one of the initiators of the St. Tecla European School on Experimental Nuclear Astrophysics (ESENA, 9 editions so far) and of the European Network of Nuclear Astrophysics Schools (ENNAS).

But the most important achievement is in my opinion that he has formed and educated a solid group of young, talented and ambitious people. He formed and lead them with "a velvet glove on an iron fist". They have competence and dynamism and they have jobs! They teach to a large part in Sicily, work in Catania and all over the world's laboratories, some are in the rest of the world. Claudio was instrumental in these!

Congratulation Claudio and good health in the years that come and success in all activities you'll chose to embrace!

The author acknowledges that many of the above have been learned from working with many in the audience, in particular with the Texas A\&M University group at the Cyclotron Institute and with the Catania group. Financial support through the grants NAIRIB C02-FAIR and PN 16390102 of the Romanian Ministry of Research and Innovation made this conference participation possible.

\section{References}

1. G. Baur, C. A. Bertulani and H. Rebel, Nucl. Phys. A458, 188 (1986)

2. A. M. Mukhamedzhanov et al. Phys Rev C 56, 1302 (1997)

3. L. Trache et al. Phys Rev C 67, 062801(R) (2003)

4. Azhari et al. Phys. Rev. Lett. 82, 3960 (1999)

5. A. Banu et al, Phys. Rev. C 79, 025805 (2009)

6. R.E. Tribble et al., Rep. Prog. Phys. 77, 106901 (2014)

7. C. R. Brune et al., Phys. Rev. Lett. 83, 4025 (1999)

8. M. L. Avila et al., Phys. Rev. Lett. 114, 071101 (2015)

9. L. Trache et al, Phys. Rev. Lett. 87, 271102 (2001); Phys. Rev. C 69, 032802 (2004)

10. A. Banu et al., Phys. Rev. C 84, 015803 (2011)

11. A. Saastamoinen et al. Phys. Rev. C 83, 045808 (2011)

12. E. Pollacco et al, Nucl. Instr. Meth. A 723, 102 (2013)

13. A. Saastamoinen et al., Nucl. Instr. Meth. B 376, 357 (2016)

14. BT Roeder et al., Nucl. Instr. Meth. A 634, 71 (2011)

15. M. McCleskey et al. Phys. Rev. C 89, 044605 (2014)

16. S. Cherubini et al. Phys. Rev. C 92, 015805 (2015)

17. R.G. Pizzone et al., Eur. Phys. J. A, 52:24 (2016)

18. L. Trache, in $4^{\text {th }}$ Eurisol User Group Topical Meeting, July 1-3, 2013, Krakow, Poland, http://www.eurisol.org/usergroup/?p=407, p. 101

19. S. Almaraz-Calderon et al., Phys. Rev. Lett. 119, 072701 (2017). 